\title{
Acute medical unit: experience from a tertiary healthcare institution in Singapore
}

Wei-Ping Goh $\underline{G}^{1}$, MBBS, MBA, Hui Fen $\underline{\operatorname{Han}}^{2}$, BSc(Hons), Uma Chandra Segara ${ }^{3}$, BSc, Geraldine $\underline{B a i r d}^{1}, \mathrm{MSN}$ Aisha Lateef ${ }^{1,4}$, MRCP, FAMS

\begin{abstract}
Singapore's healthcare system is under strain from the rising demands of an increasing and ageing population, resulting in delayed specialist care for patients presenting to the emergency department and requiring admission. Acute assessment units have been developed elsewhere but are not well established in local healthcare. Our institution extended our acute medical team to form an acute medical unit (AMU), in which focused internist-led teams are stationed on site to rapidly assess and re-triage patients. All patients (excluding those with very complex conditions) are admitted to the AMU and managed by internists who provide holistic, patient-centric care with better ownership, improved efficiency and less fragmentation. Patients can receive timely access to medical interventions and stable patients can benefit from early supported discharge, anchored by the nursing, allied health and transitional care teams. Given the ageing patient population with multiple comorbidities, this integrated model with exceptional outcomes is highly suitable for Singapore.
\end{abstract}

Keywords: acute medical unit, internist-led, Singapore

\section{INTRODUCTION}

Healthcare systems worldwide are seeing an increasing number of patients who require hospitalisation, largely due to ageing populations with multiple comorbidities as well as rising expectations of care. Modern medicine has progressed rapidly, leading to the development of highly specialised fields, but a large majority of patients today suffer from multiple diseases and complex conditions that extend beyond the scope of individual medical specialties. As such, patients are often seen by multiple specialists and/or need to wait to see a specific specialist. This contributes to the fragmentation of care, diffusion of responsibility, longer waiting times for an 'appropriate' hospital bed in an 'appropriate' specialty ward and, generally, higher costs for patients and hospitals.

Globally, multiple strategies have been employed to deal with the acute care of admitted patients and to improve system efficiency. ${ }^{(1)}$ Development of acute medical units (AMUs) for rapid assessment and care has shown great benefits in some healthcare systems. ${ }^{(2-4)}$ However, this care stratum is not well established in the Singapore healthcare model. As the local context is important, solutions should be tailored to meet local needs. ${ }^{(5,6)}$

We have previously described an intervention undertaken at our institution in the form of a virtual inpatient ward in the emergency department (ED), termed the acute medical team (AMT). ${ }^{(7)}$ In brief, a collaborative project between the Division of General Medicine, National University Hospital (NUH), Singapore, and the ED provided early definitive care to boarders waiting for beds at the division. Our earlier study showed improved patient outcomes and resource utilisation via this model of care from April 2013 to January 2015. Higher rates of early discharge (without significant differences in the readmission rates) and shorter length of stay were noted in the AMT patients, who were also more likely to be admitted into a ward specialising in their condition and have a smaller bill size compared to nonAMT patients.

We were encouraged by the outcomes from the AMT model, which were not just similar but better than those from the traditional model of care provided in the physical wards. We hypothesised that rapid assessment, investigations and treatment offered by the AMT within the critical first few hours of admission were crucial for better outcomes. Hence, it was decided to expand the model to all medical patients being admitted through the ED at our institution and convert the AMT to an AMU. In this paper, we describe the process, outcomes and learning points from the $\mathrm{AMU}$ development initiative at our institution.

\section{OPPORTUNITY TO ESTABLISH ACUTE MEDICAL UNIT}

In early 2015, our institution was faced with a challenge when a neighbouring hospital needed to scale down its services in preparation to move to a new campus. ${ }^{(8)}$ As our institution was in close proximity to this hospital and shared the same patient catchment area, we expected an unprecedented increase in patient load during the period of transition. This presented an opportunity as well as an urgent need for the hospital to scale up the working model of the AMT. The team had extensive discussions with all stakeholders, including hospital leaders, clinical departments and allied health teams. Together, they reviewed and restructured workflows to address the expected higher patient load by improving efficiency.

In March 2015, the AMT was transformed from a virtual ward to a dedicated physical area in close proximity to the ED,

\footnotetext{
${ }^{1}$ University Medicine Cluster, National University Hospital, ${ }^{2}$ Clinical Operations, Woodlands Health Campus, ${ }^{3}$ Emergency Medicine Department, National University Hospital, ${ }^{4}$ Department of Medicine, NUS Yong Loo Lin School of Medicine, National University of Singapore, Singapore

Correspondence: Dr Goh Wei-Ping, Principal Resident Physician, University Medicine Cluster, National University Hospital, 1 E Kent Ridge Road, Singapore 119074. wei_ping_goh@nuhs.edu.sg
} 
refurbished to become an AMU. We developed a new care delivery system: all patients that the ED assigned for admission to the Department of Medicine were admitted to this dedicated internist-led unit (Fig. 1). The AMU allowed delivery of acute medical care in a single location with integration and concentration of rapid diagnostic and therapeutic interventions that could impact patient care. During the initial rollout, the AMU had a functional capacity of 40 patients to allow testing and refining of the new workflows. During the period when the other hospital closed down and our patient numbers rose, the AMU was expanded to care for up to 75 patients. The steady state current capacity of the unit is 40 patients.

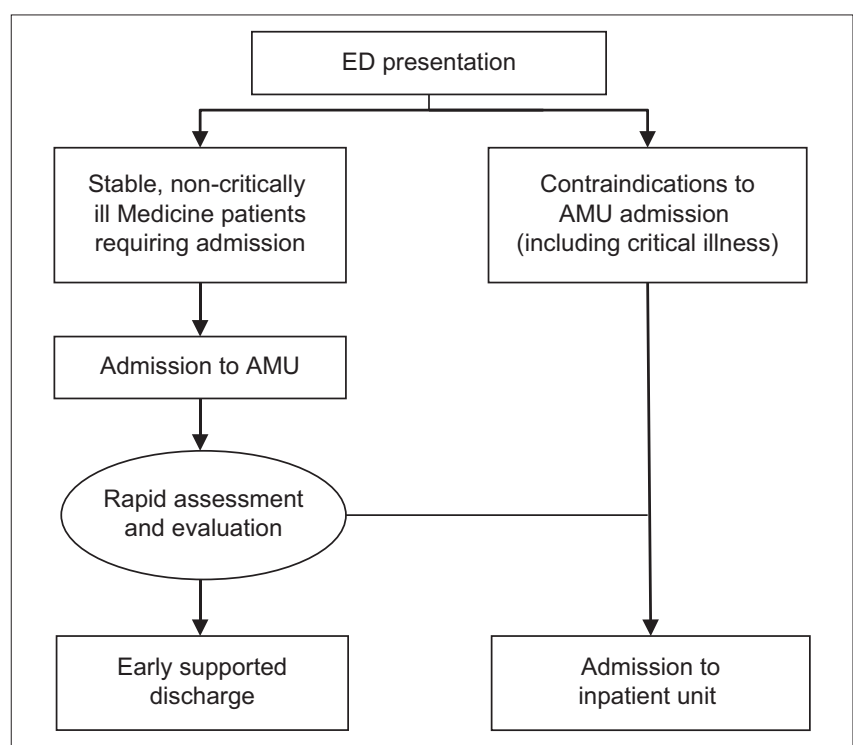

Fig. 1 Flowchart shows patient selection from presentation to the emergency department (ED). AMU: acute medical unit; Medicine: Department of Medicine

\section{INTERNIST-LED MODEL OF CARE}

The AMU continued to use an internist-led model of care, which differs from the existing specialist-led care model (Fig. 2). Historically, after initial review and stabilisation at the ED, patients were admitted to the various inpatient wards under different specialties. However, with the ageing population and increasing multi-morbidity in Singapore, many patients suffer from multiple and complex conditions that extend beyond the scope of individual medical specialties. Furthermore, a large group of medical patients present with undifferentiated complaints, and an initial assessment by an internist would be beneficial to further streamline their management. In the AMU model, all medical patients were admitted to a single location for further assessment before they were discharged or further triaged for subspecialty transfer to an appropriate inpatient bed. While the maximum permitted stay in AMU was not dictated, clinicians generally did not keep patients in the AMU for more than 48 hours. Patients who were identified as suitable for fast-track discharge were kept in the AMU for investigations and observation, while the rest were admitted to the wards for further management by the specialty teams after an initial period of rapid assessment and evaluation.

\section{Resource planning}

When the $\mathrm{AMU}$ was first rolled out, both the medical and nursing teams needed to be reinforced to meet increased demands from both the larger volume and a wider case mix. Additional medical teams were deployed to the AMU, each comprising one consultant, 1-2 senior residents and 1-2 residents. Consultants from other subspecialties with an interest in holistic, integrated, patient-centric care were invited to join the team as internists. These internists were readily supported and advised by teams

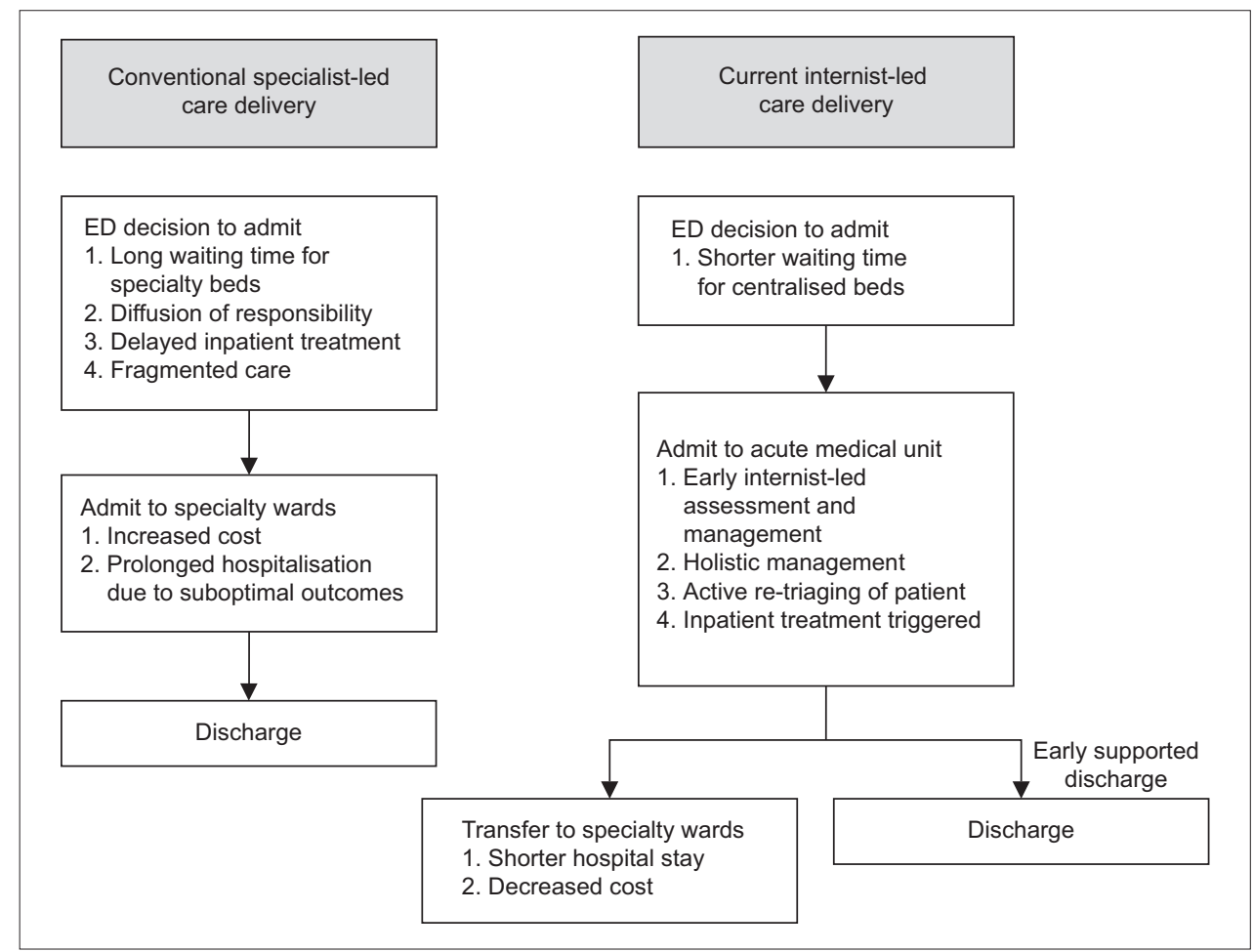

Fig. 2 Comparison of the conventional specialist-led and internist-led models of care. ED: emergency department 


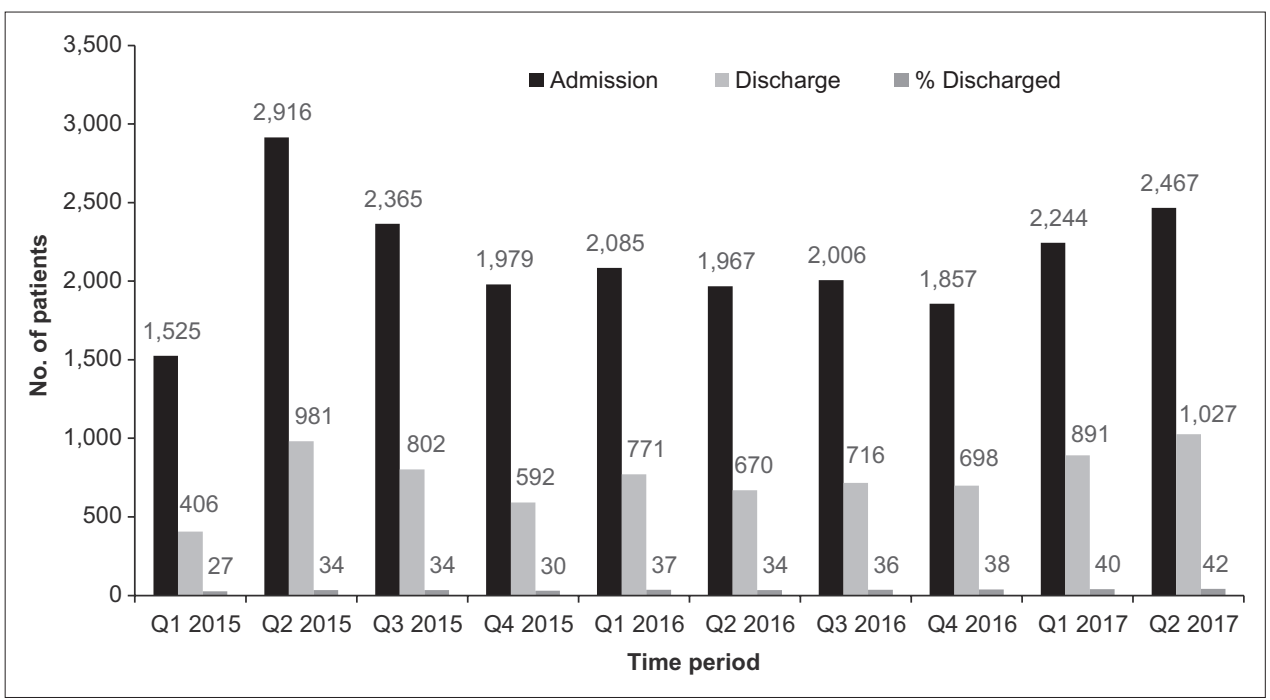

Fig. 3 Bar chart shows admissions and discharges from the acute medical unit.

from other subspecialties, and helped to coordinate the care of the patients. Nurses working in the AMU were primarily from the ED. They underwent further training to be equipped with additional skill sets to manage the new case mix of patients. Dedicated allied health services (i.e. from physiotherapy, occupational therapy, speech therapy, podiatry and clinical nutrition), clinical management support (i.e. transitional care team, asthma/chronic obstructive pulmonary disease/transient ischaemic attack case managers and fall prevention nurse) and community services support (i.e. care coordinators and social workers) were made available to all AMU patients. Imaging and endoscopy requests were also prioritised for patients in the AMU.

Fig. 3 shows the number of admissions and direct discharges from the AMU from the first quarter of 2015 to the second quarter of 2017. The surge in patient numbers in the second quarter of 2015 was due to the decrease in the inpatient load from our neighbouring hospital to facilitate its move in June 2015. After that, direct discharges from the AMU were consistently maintained above $30 \%$, reaching a high of $42 \%$ in the second quarter of 2017. The top 20 discharge diagnoses from the AMU during the same period are listed in Table I. These included common symptom-based diagnoses such as giddiness, dizziness, chest pain and headache, as well as disease diagnoses such as gastritis, pneumonia and gastroenteritis.

\section{BENEFITS OF THE MODEL}

The AMU internist-led integrated model of care involves fewer specialists across the continuum from inpatient to post-discharge care. The internists are motivated specialists from internal medicine or another subspeciality within the Department of Medicine, or specialists who had undergone dual training (in internal medicine and a subspecialty), with a passion for integrated care. They provide holistic, patient-centric care with better overall ownership of the patients, improved efficiency and less fragmentation of care.

The AMU model of care also addresses conditions in which management plans can be expedited through having rapid access to
Table I. Top 20 discharge diagnoses from the acute medical unit.

\begin{tabular}{|ll|}
\hline Diagnosis & $\%$ \\
\hline Dizziness and giddiness & 9.42 \\
\hline Gastritis & 6.98 \\
\hline Diabetes mellitus & 6.02 \\
\hline Upper respiratory infection & 4.69 \\
\hline Pneumonia & 4.51 \\
\hline Chest pain & 3.37 \\
\hline Gastroenteritis and colitis & 3.34 \\
\hline Head injury & 3.16 \\
\hline Headache & 3.04 \\
\hline Asthma & 2.80 \\
\hline Constipation & 2.65 \\
\hline Urinary tract infection & 2.02 \\
\hline Cellulitis & 1.99 \\
\hline Hypertension & 1.93 \\
\hline COPD & 1.84 \\
\hline Syncope & 1.77 \\
\hline Epilepsy & 1.65 \\
\hline
\end{tabular}

COPD: chronic obstructive pulmonary disease

investigations. For example, our ED has its own diagnostic imaging room with a dedicated computed tomography $(\mathrm{CT})$ machine that has a lower utilisation rate compared to the machines used by inpatient wards, due to the need for some redundancy so as to allow rapid availability of available imaging slots for emergencies. The close proximity of the AMU and ED makes it much easier to coordinate imaging for $\mathrm{AMU}$ patients in need of urgent radiography or $\mathrm{CT}$, and allows for better utilisation of the machine in the ED without competing with inpatients for appointment slots.

The AMU model is supported by discharge enablers, including timely same-day allied health assessment reviews, follow-up by the transitional care team and rapid access to clinic appointments. Discharge of patients directly from the AMU meant that those with mild ailments are not admitted to the inpatient wards. This allows the specialty inpatient clinical teams to concentrate on the care of patients who truly need advanced tertiary care. 
The concentration of medical admissions in one location also helps the hospital to rationalise resource allocation. For example, the high number of new admissions with new medication orders justified the need for an on-site pharmacist in the AMU. Pharmacists in inpatient wards are usually not on site, but located at satellite pharmacies a short distance away. Traditionally, this creates challenges, as pharmacists have to contact clinicians (often via phone calls or short message service) for clarification of medication orders and vice versa. Having on-site pharmacists facilitates processes such as timely medication reviews and reconciliation, consults on medication doses and adjustments, and discharge medication preparation.

The AMU takes over the care of patients immediately after the decision to admit is made by the ED team. This allows the ED to focus on other patients who may need more immediate and urgent medical attention, and removes the burden of looking after patients who are waiting for beds. Hence, the AMU has led to better containment and significantly reduced scatter of medical patients, especially to surgical wards. This translates into more beds available for surgical, cardiac and other departments, despite the heavy patient load and high bed occupancy rates. The capacity generated for other departments to function and continue their work also enables overall improved and smoother running of the hospital.

The AMU is a rich resource with bountiful educational opportunities. It serves as a good learning ground for residents and medical students with its remarkable case mix and exposure to acute medicine. The posting is much sought after by residents, medical students and nursing students in our institution. Trainees can be exposed to a wider spectrum of medical conditions and are better trained in holistic patient care, managing the whole person instead of the individual disease. The early supported discharge culture also provides good learning in systems-based practice, with appropriate utilisation of healthcare resources for safe yet efficient patient care in hospitals as well as community settings. The AMU initiative has allowed us to identify patient groups and diagnoses that would benefit from the new model of care and aid in resource planning for the future. With the recent move to increase the generalist pool in Singapore, its development is timely and necessary in an ageing population with multiple medical problems. In addition, learning points from managing the AMU are guiding us in the development of workflows and protocols to help manage common medical conditions that require short inpatient stays. Increasingly, our allied health colleagues, such as advanced practice nurses, are getting more involved in the formulation of care plans for our patients as part of a care team. Advanced practice nurses have been invaluable and their roles can be further guided by these workflows so that they can assume greater responsibilities in patient care. Other healthcare workers, including clinicians and allied health teams who previously worked in specialty-specific areas, are also getting upskilled with cross-department training to prepare them for the patient mix as we move towards a generalist model of care.

\section{CONCLUSION}

The formation of the AMU has allowed the provision of care in a single location during the critical hours following initial assessment and stabilisation at the ED. Being a focal point of acute activities, the AMU has allowed the department, in consultation with hospital leadership, to rationalise resource allocation. The AMU has shown sustained benefits, providing early definitive care to a large fraction of medical patients. Employing and integrating resources such as advanced practice, physiotherapy and home care nurses, we were able to avoid ward admission for one-third of patients, which led to thousands of bed days saved within the short period of the $\mathrm{AMU}$ rollout.

In conclusion, the AMU has shown the capability to deliver focused, efficient and early definitive care to large numbers of patients, as well as sustain a very heavy patient load during the move of the other hospital, which was truly a testament to the viability of the unit and model of care. The AMU model has the potential to improve and reshape the healthcare system in Singapore.

\section{REFERENCES}

1. Elder E, Johnston AN, Crilly J. Review article: systematic review of three key strategies designed to improve patient flow through the emergency department. Emerg Med Australas 2015; 27:394-404.

2. Vork JC, Brabrand M, Folkestad L, et al. A medical admission unit reduces duration of hospital stay and number of readmissions. Dan Med Bull 2011; 58:A4298.

3. Li JY, Yong TY, Bennett DM, et al. Outcomes of establishing an acute assessment unit in the general medical service of a tertiary teaching hospital. Med J Aust 2010; 192:384-7.

4. Scott I, Vaughan L, Bell D. Effectiveness of acute medical units in hospitals: a systematic review. Int J Qual Health Care 2009; 21:397-407.

5. van Galen LS, Lammers EM, Schoonmade LJ, et al. Acute medical units: the way to go? A literature review. Eur J Intern Med 2017; 39:24-31.

6. Jenkins PF, Barton LL, McNeill GB. Contrasts in acute medicine: a comparison of the British and Australian systems for managing emergency medical patients. Med J Aust 2010; 193:227-8.

7. Lateef $\mathrm{A}$, Lee $\mathrm{SH}$, Fisher DA, et al. Impact of inpatient care in emergency department on outcomes: a quasi-experimental cohort study. BMC Health Serv Res 2017; 17:555.

8. Grosse S. Ng Teng Fong General Hospital to open on Jun 30 [online]. Channel NewsAsia 2015 Apr 29. Available at: http://archive.is/UX4o8. Accessed August 27, 2018. 